\title{
Near-Heisenberg-Limited Atomic Clocks in the Presence of Decoherence
}

\author{
J. Borregaard* and A. S. Sørensen ${ }^{\dagger}$ \\ QUANTOP, The Niels Bohr Institute, University of Copenhagen, Blegdamsvej 17, DK-2100 Copenhagen $\emptyset$, Denmark
}

(Received 12 April 2013; published 27 August 2013)

\begin{abstract}
The ultimate stability of atomic clocks is limited by the quantum noise of the atoms. To reduce this noise it has been suggested to use entangled atomic ensembles with reduced atomic noise. Potentially this can push the stability all the way to the limit allowed by the Heisenberg uncertainty relation, which is denoted the Heisenberg limit. In practice, however, entangled states are often more prone to decoherence, which may prevent reaching this performance. Here we present an adaptive measurement protocol that in the presence of a realistic source of decoherence enables us to get near-Heisenberg-limited stability of atomic clocks using entangled atoms. The protocol may thus realize the full potential of entanglement for quantum metrology despite the detrimental influence of decoherence.
\end{abstract}

DOI: 10.1103/PhysRevLett.111.090801

Atomic clocks provide some of the most accurate time measurements in physics. One of the main limitations to the stability of atomic clocks is the quantum noise of the atoms, which leads to the standard quantum limit where the stability scales as $1 / \sqrt{N}$ with $N$ being the number of atoms $[1,2]$. To overcome this noise it has been suggested to use entangled states with reduced atomic noise [3-7]. Ultimately this may lead to a stability at the Heisenberg limit where the resolution scales as $1 / N$, and recently the first proof of principle experiments have demonstrated these concepts experimentally [8-13]. In practice, however, entangled states are often more prone to decoherence, and to fully assess the advantage it is essential to study the performance in the presence of decoherence [14]. In Ref. [5] it was proven that entanglement can be used to improve the long-term stability of atomic clocks in the presence of the dominant practical source of decoherence, but the improvement identified was rather limited. Here we show that it is possible to obtain a large improvement in the stability of the clock by combining entanglement with an adaptive measurement protocol (inspired by Refs. $[15,16]$ ). With our adaptive measurement protocol the entangled states are not more sensitive to the decoherence than disentangled states [cf. Fig. 1(b)]. As a consequence the long-term stability of the atomic clock can be improved almost to the Heisenberg limit even in the presence of decoherence.

Many atomic clocks are operated by locking a local oscillator (LO) to an atomic transition via a feedback loop. The feedback is typically based on a measurement of the LO frequency offset $\delta \omega$ compared to the atomic transition through Ramsey spectroscopy [17]. Here the atoms are first prepared in one of the two clock states by, e.g., a laser pulse. During the Ramsey sequence the atoms interact with the LO field. This interaction consists of three parts: first the atoms are subject to a near-resonant $\pi / 2$ pulse from the LO followed by the Ramsey time $T$ of free evolution, and finally another near-resonant $\pi / 2$ pulse is
PACS numbers: 06.30.Ft, 03.65.Ud, 03.65.Yz, 06.20.Dk

applied to the atoms. During the free evolution the LO acquires a phase $\delta \phi=\delta \omega T$ relative to the atoms. Due to the last $\pi / 2$ pulses this phase can be measured as a population difference between the two clock levels. $\delta \omega$ can thus be estimated from the measurement and used for a feedback that steers the frequency of the LO to the atomic frequency. The stability of the clock will improve with $T$ since a longer $T$ improves the relative sensitivity of the frequency measurement. For current atomic fountain clocks, $T$ is limited by gravity and can hardly be varied [18]. Here on the other hand we consider trapped particles, where $T$ can be increased until it is limited by the decoherence in the system [19-21]. The long-term stability thus depends on the nature of the decoherence.

To take decoherence into account Ref. [14] considered single atom dephasing. For this model Ref. [14] showed that entanglement cannot improve the stability of atomic clocks considerably (although an improvement is possible
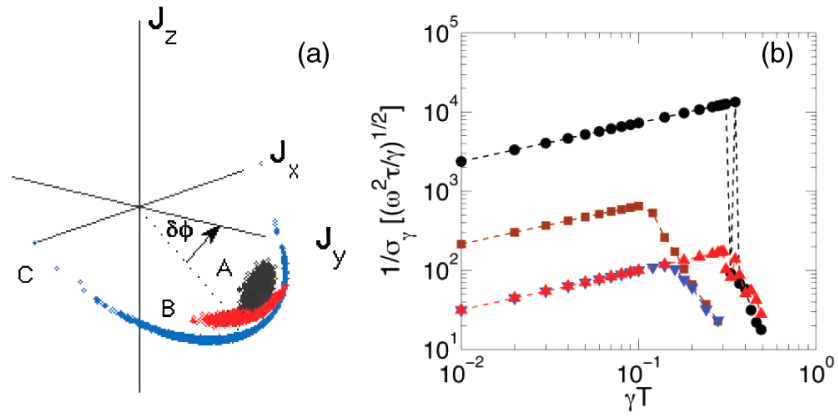

FIG. 1 (color online). (a) The atomic state just before the measurement of $J_{z}$ for (A) uncorrelated atoms, (B) moderately squeezed atoms, and (C) highly squeezed atoms. (b) Stability as a function of the Ramsey time $(\gamma T)$ for $N=10^{5} . \mathbf{Q},(\boldsymbol{\nabla})$ is the conventional protocol of Ref. [5] for optimal squeezing (uncorrelated) atoms while $\boldsymbol{O},(\mathbf{\Delta})$ is the adaptive protocol for optimal squeezing (uncorrelated) atoms. The adaptive protocol allows for $\gamma T \sim 0.3$ while the conventional protocol only allows for $\gamma T \sim 0.1$ (see the Supplemental Material [32]). 
for non-Markovian noise [22]). A more realistic model of the decoherence was described in Ref. [5] where the primary noise source is the frequency fluctuations of the LO [23]. In this work a small improvement in the longterm stability, scaling as $\sim N^{1 / 6}$, was identified for entangled atoms. Here we use the same decoherence model and disregard any decoherence of atoms, to show that entanglement and adaptive measurements may improve the performance and give near-Heisenberg-limited atomic clocks. Although the assumption of negligible atomic decoherence may be hard to fulfill for the highly entangled states considered here, our results highlight that there is no fundamental obstacle to reaching the Heisenberg limit. Another approach to increase the stability is to increase $T$ [24-26]. In particular Ref. [26] increases $T$ through a measurement protocol highly related to ours. However that work considers a scenario where the clock is limited by technical noise so that a direct comparison with our results is not possible. Which protocol is advantageous is thus an open question beyond the scope of this Letter.

We consider an ensemble of $N$ two-level atoms, which we model as a collection of spin- $1 / 2$ particles with total angular momentum $\vec{J}$. The angular momentum operators $\hat{J}_{x, y, z}$ give the projections of $\vec{J}$ on the $x, y$, and $z$ axis. The atoms are initially pumped to have a mean spin along the $z$ axis, $\left\langle\hat{J}_{x}\right\rangle=\left\langle\hat{J}_{y}\right\rangle=0$. After the Ramsey sequence the Heisenberg evolution of $\hat{J}_{x}, \hat{J}_{y}$, and $\hat{J}_{z}$ is $\hat{J}_{1}(\delta \phi)=\hat{J}_{x}, \quad \hat{J}_{2}(\delta \phi)=\sin (\delta \phi) \hat{J}_{y}-\cos (\delta \phi) \hat{J}_{z}, \quad$ and $\hat{J}_{3}(\delta \phi)=\cos (\delta \phi) \hat{J}_{y}+\sin (\delta \phi) \hat{J}_{z}$. At the end of the Ramsey sequence $\hat{J}_{3}$ is measured and used to estimate $\delta \phi$. The $\hat{J}_{y}$ term in $\hat{J}_{3}$ results in the so-called projection noise in the phase estimate $\sim \Delta J_{y} /\left|\left\langle J_{z}\right\rangle\right|$. For uncorrelated atoms $\Delta \hat{J}_{y} \Delta \hat{J}_{x}=\left\langle\hat{J}_{z}\right\rangle / 2 \approx N / 4$ and the projection noise causes the stability of the clock to scale as $\sim 1 / \sqrt{N}$. For a spin squeezed state [27] the variance of $\hat{J}_{y}$ is reduced to obtain a better phase estimate. Such a spin squeezed state is depicted in Fig. 1(a), which shows how the spin squeezed state looks like a "flat banana" on the Bloch sphere. The more we squeeze, the longer and more narrow the banana is and significant extra noise is added to the mean spin direction. For a phase estimate based on a direct measurement of $\hat{J}_{3}$ this gives an additional noise term $\sim \delta \phi \Delta \hat{J}_{z} /\left|\left\langle J_{z}\right\rangle\right|$. This extra noise limited the performance in Ref. [5] if strongly squeezed states were used. We avoid this problem by using an adaptive scheme with weak measurements to make a rough estimate of $\delta \phi$ and then rotate the spins of the atoms such that the mean spin is almost along the $y$ axis. The flat banana depicted in Fig. 1(a) will then lie in the $x y$ plane and this will decrease the noise from $\Delta \hat{J}_{z}$ in subsequent measurements (see Fig. 2). Having eliminated the noise from $\Delta \hat{J}_{z}$ we can allow strong squeezing in $\Delta \hat{J}_{y}$ and obtain nearHeisenberg-limited stability.

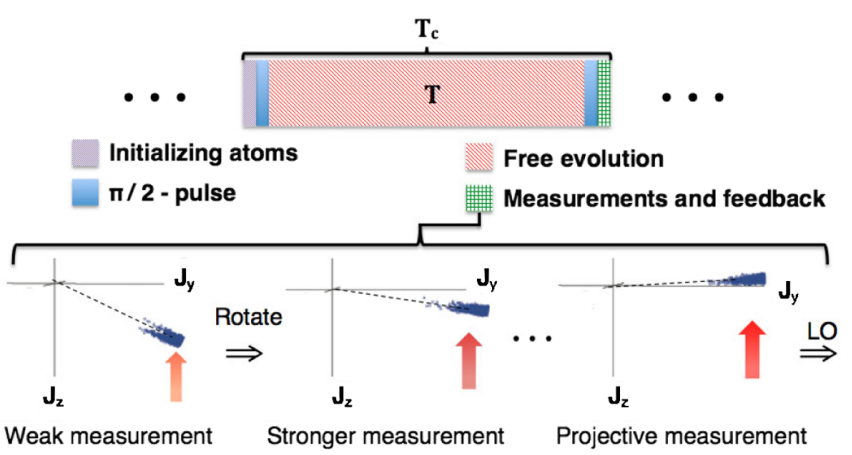

FIG. 2 (color online). Operation of an atomic clock. A clock cycle of duration $T_{c}$ starts with initializing the atoms and ends with the measurements and feedback on the LO. The bottom part of the figure shows the adaptive protocol consisting of a series of weak measurements with intermediate feedback. The feedback seeks to rotate the atomic state to have mean spin almost along the $y$ axis before the final projective measurement and subsequent feedback on the LO.

The operation of the clock consists of repeating the clock cycle illustrated in Fig. 2. The total cycle duration $T_{c}$ will be larger than the period of free evolution due to the time spent on preparation and measurement of the atoms, and this dead time introduces Dick noise to the stability [28]. To focus on the atomic noise we assume that the dead time is negligible $\left(T_{c} \sim T\right)$ so that we can ignore the Dick noise. (Alternatively some clock based measurements can also be constructed which are immune to Dick noise [29-31]). This assumption is further discussed in the Supplemental Material [32]. We discretize time in the number of clock cycles $(k)$ such that at time $t_{k}=k T$ the frequency correction $\Delta \omega\left(t_{k}\right)=-\alpha \delta \phi^{e}\left(t_{k}\right) / T$ is applied to the LO where $\alpha$ sets the strength of the feedback loop and $\delta \phi^{e}\left(t_{k}\right)$ is the estimate of the accumulated phase $\delta \phi\left(t_{k}\right)$ between time $t_{k-1}$ and $t_{k}$. The frequency offset of the LO at time $t_{k}$ is then $\delta \omega\left(t_{k}\right)=\delta \omega_{0}\left(t_{k}\right)+\sum_{i=1}^{k} \Delta \omega\left(t_{i}\right)$, where $\delta \omega_{0}\left(t_{k}\right)$ is the frequency fluctuation of the unlocked LO. The mean frequency offset after running for a period $\tau=l T(l \gg 1)$ is (see the Supplemental Material [32])

$$
\delta \bar{\omega}(\tau)=\frac{1}{l} \sum_{k=1}^{l} \frac{\delta \phi\left(t_{k}\right)-\delta \phi^{e}\left(t_{k}\right)}{T},
$$

resulting in the long-term stability of the atomic clock:

$$
\begin{aligned}
\sigma_{\gamma}(\tau) & =\left\langle(\delta \bar{\omega}(\tau) / \omega)^{2}\right\rangle^{1 / 2} \\
& =\sqrt{\frac{1}{\tau \omega^{2}}}\left(\frac{1}{l} \frac{\left\langle\left(\sum_{k=1}^{l} \delta \phi\left(t_{k}\right)-\delta \phi^{e}\left(t_{k}\right)\right)^{2}\right\rangle}{T}\right)^{1 / 2} .
\end{aligned}
$$

We initially assume that the phase offset of the unlocked LO $\delta \phi_{0}$ is due to frequency fluctuations in the LO with a white noise spectrum. Later we will also consider the case where the fluctuations have a $1 / f$ spectrum. For white noise we have $\left\langle\delta \phi_{0}^{2}\right\rangle=\gamma T\left(\left\langle\delta \phi_{0}\right\rangle=0\right)$ where $\gamma$ is a 
parameter characterizing the fluctuations. In the limit $\alpha \ll 1$, the phases are uncorrelated (see the Supplemental Material [32]) such that $\sigma_{\gamma}(\tau)=$ $\sqrt{\gamma / \tau \omega^{2}}\left(\left\langle\left(\delta \phi_{0}-\delta \phi^{e}\right)^{2}\right\rangle / \gamma T\right)^{1 / 2}$. This expression shows that for fixed $\gamma$ and $\tau$ the stability of the clock only depends on how precisely we can estimate $\delta \phi_{0}$.

We now describe our adaptive measurements in detail. Our weak measurements are based on the strategy developed and demonstrated in Refs. $[10,11,33,34]$ where a light field dispersively interacts with the spin and is subsequently measured. This is described by a Hamiltonian $H_{\text {int }}=-\chi_{1} \hat{J}_{3} \hat{X}_{1}$ where $\chi_{1}$ is the interaction strength and $\hat{X}_{1}$ is the canonical position operator of the light [35-37]. The measurement results in a rotation around $\hat{J}_{3}$ described by the rotation matrix $\mathbf{R}_{3}\left(\hat{\Pi}_{1}\right)$, where $\hat{\Pi}_{1}=\Omega_{1} \hat{X}_{1} . \Omega_{1}=$ $\chi_{1} \mu_{1}$ is the measurement strength and $\mu_{1}$ is the measurement time. The canonical momentum operators of the light before $\left(\hat{P}_{1}\right)$ and after $\left(\hat{P}_{1}^{\prime}\right)$ the interaction are then related by $\hat{P}_{1}^{\prime}=\hat{P}_{1}-\Omega_{1} \hat{J}_{3}$. $\hat{P}_{1}^{\prime}$ is measured using homodyne detection [38] and the phase is estimated as $\delta \phi_{1}^{e}=$ $-\left(\beta_{1} \hat{P}_{1}^{\prime}\right) / \Omega_{1}\left\langle\hat{J}_{z}\right\rangle$, where the factor $\beta_{1}$ is found from minimizing $\left\langle\left(\delta \phi_{0}-\delta \phi_{1}^{e}\right)^{2}\right\rangle$. Based on the phase estimate we rotate the spin of the atoms around $\hat{J}_{1}$ in order to compensate for the extra noise added $\left(\Delta J_{z}\right)$ by the spin squeezing. This is described by a rotation matrix $\mathbf{R}_{1}\left(\delta \phi_{1}^{e}\right)$. The process can be iterated such that after $n-1$ weak measurements the Heisenberg evolution of the original operators $\left(\hat{J}_{1}, \hat{J}_{2}, \hat{J}_{3}\right)$ is

$$
\begin{aligned}
\left(\begin{array}{c}
\hat{J}_{1} \\
\hat{J}_{2} \\
\hat{J}_{3}
\end{array}\right)_{n}= & \mathbf{R}_{1}\left(\delta \phi_{n-1}^{e}\right) \mathbf{R}_{3}\left(\hat{\Pi}_{n-1}\right) \ldots \mathbf{R}_{1}\left(\delta \phi_{1}^{e}\right) \\
& \times \mathbf{R}_{3}\left(\hat{\Pi}_{1}\right)\left(\begin{array}{c}
\hat{J}_{1} \\
\hat{J}_{2} \\
\hat{J}_{3}
\end{array}\right) .
\end{aligned}
$$

The final measurement is assumed to be a projective measurement and the final phase estimate $\delta \phi_{n}^{e}$ is thus $\delta \phi_{n}^{e}=$ $\beta_{n} \hat{J}_{3, n} /\left\langle\hat{J}_{z}\right\rangle$. The factors of $\beta_{i}$ in the phase estimates are found by minimizing $\left\langle\left(\delta \phi_{0}-\sum_{j=1}^{i} \delta \phi_{j}^{e}\right)^{2}\right\rangle$ with respect to $\beta_{i}$ after each measurement. The final estimate of $\delta \phi_{0}$ at the end of the measurement sequence is $\delta \phi^{e}=\sum_{i=1}^{n} \delta \phi_{i}^{e}$, where $\delta \phi_{i}^{e}$ is the phase estimate after the $i$ th measurement.

We will now show semianalytically that the measurement strategy in Eq. (3) allows for near-Heisenberg-limited stability. For simplicity we set all $\beta_{i}=1$ in our analytical calculations. After $j$ weak measurements the difference between the true phase and the estimated phase $\delta \Phi_{j}$ is

$$
\delta \Phi_{j}=\delta \phi_{0}-\sum_{i=1}^{j} \delta \phi_{i}^{e}=\delta \phi_{0}-\sum_{i=1}^{j-1} \delta \phi_{i}^{e}-\delta \phi_{j}^{e} .
$$

Using Eq. (3) to get an expression for $\delta \phi_{j}^{e}$ and the fact that $\delta \Phi_{j-1}=\delta \phi_{0}-\sum_{i=1}^{j-1} \delta \phi_{i}^{e}$, we can express the phase error as

$$
\begin{aligned}
\delta \Phi_{j} \approx & \delta \Phi_{j-1}\left(1-\hat{J}_{z} /\left\langle\hat{J}_{z}\right\rangle\right) \\
& -\left(\hat{J}_{y}+\delta \hat{J}_{3, j}-\hat{P}_{j} / \Omega_{j}\right) /\left\langle\hat{J}_{z}\right\rangle,
\end{aligned}
$$

where we have assumed $\delta \Phi_{j-1} \ll 1$. The first term in Eq. (5) gives a contribution $\sim \delta \Phi_{j-1} \Delta J_{z} /\left\langle\hat{J}_{z}\right\rangle$ to $\sigma_{\gamma}(\tau)$ from the noise in the mean spin direction as discussed previously. Note that this term is proportional to the phase estimation error at the previous measurement stage, since it depends on how well the banana in Fig. 1(a) is rotated into the $x y$ plane. For a useful adaptive protocol $\delta \Phi_{j-1}$ gets smaller for growing $j$ and the noise that enters through $\Delta \hat{J}_{z}$ is reduced. The last terms in Eq. (5) gives the noise from $\Delta \hat{J}_{y}$, the accumulated backaction of the previous measurements $\left(\left\langle\delta J_{3, j}^{2}\right\rangle\right)$, and the noise from the incoming light in the measurement $\left(\Delta \hat{P}_{j}^{2}=\left\langle\hat{P}_{j}^{2}\right\rangle\right)$.

The stronger a measurement is, the less noise is added through $\Delta \hat{P}_{j}^{2} / \Omega_{j}^{2}$ since the measurement is more precise. Any imprecision $\Delta \hat{P}_{i<j}^{2} / \Omega_{i<j}^{2}$ from previous measurements is contained in $\delta \Phi_{j-1}$ and is corrected for in the subsequent stages of the protocol, which estimate how well we corrected the phase in previous measurements. This means that we can initially work with weak measurements, which only give a rough estimate since later stronger measurements correct for the imprecision in the initial measurements.

The accumulated backaction noise $\delta \hat{J}_{3, j}$ originates from the disturbance caused by the measurements. The measurements add noise in $\hat{J}_{1}, \hat{J}_{2}$, which is mixed into $\hat{J}_{3}$ when the atomic state is rotated to have mean spin almost along the $y$ axis. From Eq. (3) the dominant term in $\delta \hat{J}_{3, j}$ is found to be $\delta \hat{J}_{3, j} \sim \sum_{i=1}^{j-1} \delta \phi_{i}^{e} \Omega_{i} \hat{X}_{i} \hat{J}_{x}$ (see the Supplemental Material [32]). The stronger a measurement is, the more noise is added to the stability. For a useful adaptive protocol however, $\delta \phi_{i}^{e}$ gets smaller for growing $i$, which means that the $i$ th measurement can be stronger than the previous $(i-1)$ th measurements without adding more noise to the stability.

Above we have argued that we can suppress the noise terms originating from $\Delta J_{z}, \Delta \hat{P}_{j}$, and $\delta \hat{J}_{3, j}$ using an adaptive protocol with weak initial measurements. A remaining question is how well this suppression works. This is considered in detail in the Supplemental Material [32]. To be specific, we consider spin squeezed states of the form $|\psi(\kappa)\rangle=\mathcal{N}(\kappa) \sum_{m}(-1)^{m} e^{-(m / \kappa)^{2}}|m\rangle$, where $|m\rangle$ are eigenstates of $\hat{J}_{y}$ with eigenvalue $m, \mathcal{N}(\kappa)$ is a normalization constant, and the sum is from $-J$ to $J$ where $J=$ $N / 2$ is the total angular momentum quantum number. This form gives a simple family of states characterized by a single parameter $(\kappa)$, which can extrapolate between 

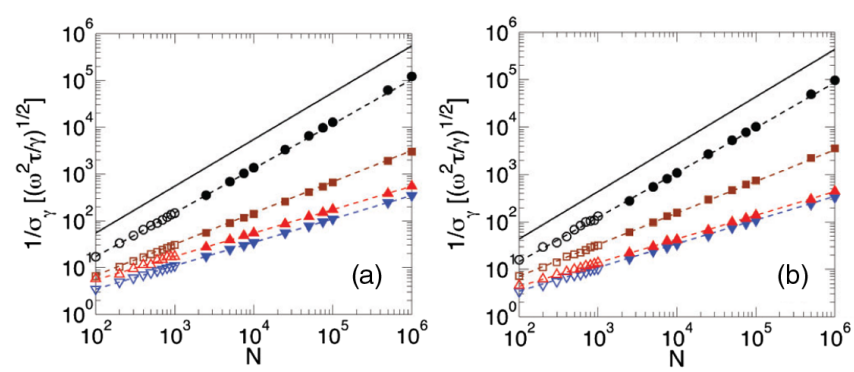

FIG. 3 (color online). Optimized stability of an atomic clock for a LO subject to (a) white noise and (b) $1 / f$ noise. $\bigcirc, \square, \triangle, \nabla$ are the full quantum simulation while $\boldsymbol{O}, \mathbf{\square}, \boldsymbol{\Delta}, \boldsymbol{\nabla}$ are the Gaussian simulation. The Gaussian simulation can be extended down to $N=100$, which gives more or less identical results to the full quantum simulation. $\bigcirc, \boldsymbol{\Theta}(\boldsymbol{\Delta}, \triangle)$ are the adaptive scheme and $\square, \boldsymbol{\square}(\nabla, \boldsymbol{\nabla})$ are the conventional protocol with (without) entanglement. The dotted lines are the analytical results and the solid line is the Heisenberg limit for the maximal Ramsey time $\gamma T=0.3$ (a) and $\gamma T=0.2$ (b).

uncorrelated states $\kappa=\sqrt{N}$ and highly squeezed states approaching the $|m=0\rangle$ Fock state $\kappa \rightarrow 0$. It may be possible to identify more optimal states [39] but this simple form is sufficient for our present purpose. We furthermore assume that the probe light has vacuum statistics. As an upper limit of the stability we find that for $n \geqslant 3 \log (N)$ weak measurements, using a spin squeezed state with $\kappa \sim \log \sqrt{N}+2$ and choosing a measurement strategy with $\Omega_{i} \sim N^{-1+i /(n+1)}$ we can suppress other noise terms so that the measurement is eventually limited by $\Delta \hat{J}_{y}$. $\sigma_{\gamma}(\tau)$ will then be $\sim(2 / N+\log \sqrt{N} / N) / \sqrt{\gamma T}$ (in units of $\sqrt{\gamma / \tau \omega^{2}}$ ) for $N \gg 1$. It is seen that for $N=10^{6}$ the upper limit of the stability will differ from the Heisenberg limit $\sigma_{\gamma}(\tau)=(1 / N) / \sqrt{\gamma T}$ by a factor of $\sim 5$.

The above upper limit shows that we can be near the Heisenberg limit, but to get the optimal stability we numerically minimized $\sigma_{\gamma}$. We simulated an atomic clock with a LO subject to white Gaussian noise for atom numbers in the range $N=100$ to $N=10^{6}$ (see the Supplemental Material [32]). For $N \leq 1000$ we simulated the full quantum evolution (we denote this as "full quantum simulation") and for $N>1000$ we approximated the probability distributions of $\hat{J}_{x, y, z}$ with Gaussian distributions with moments calculated from $|\psi(\kappa)\rangle$ in the approximation $N \gg 1$ (we denote this as "Gaussian simulation").

An example of the results is shown in Fig. 1(b), which demonstrates that there is a significant improvement by using a spin squeezed state compared to uncorrelated atoms. With the adaptive protocol, the Ramsey time can be as large for highly entangled states as for disentangled states and there is thus no difference in the relevant coherence time. Furthermore because the adaptive protocol can determine phases $\lesssim \pi$ it allows longer interrogation times $\gamma T \lesssim 0.3$ than the conventional protocol $\gamma T \lesssim 0.1$, which begins to give ambiguous results for phases $\sim \pi / 2$.
We have numerically minimized $\sigma_{\gamma}(\tau)$ in the degree of squeezing, the number of weak measurements, the Ramsey time, and the strengths of the measurements. Figure 3(a) shows the result of the optimization for both the adaptive protocol and the conventional protocol with and without squeezing. The adaptive protocol gives a significant improvement compared to using uncorrelated atoms resulting in near-Heisenberg-limited stability. The numerical calculations also agree nicely with the analytical calculations (see the Supplemental Material [32]). As noted above the adaptive protocol allows for a longer Ramsey time than the conventional protocol, which gives an improvement of roughly a factor 1.6 for uncorrelated atoms.

So far we have assumed white noise in the LO. In practice the noise of the LO is however more likely to have a nontrivial spectrum like $1 / f$ noise. We have therefore repeated the numerical optimization with $1 / f$ noise in the LO for which $\left\langle\omega(f) \omega\left(f^{\prime}\right)\right\rangle=\delta\left(f+f^{\prime}\right) \gamma^{2} / f$ and the results are shown in Fig. 3(b) (see the Supplemental Material [32]). The improvement obtained using the adaptive scheme with correlated atoms persists also for $1 / f$ noise. Again nearHeisenberg-limited stability is obtained using the adaptive protocol. The longer Ramsey time of the adaptive scheme compared to projective measurements gives an improvement of roughly a factor 1.3 for uncorrelated atoms.

In conclusion we have developed an adaptive measurement protocol which allows operating atomic clocks near the Heisenberg limit using entangled spin squeezed ensembles of atoms. These results clearly demonstrate that entanglement can be an important resource for quantum metrology. Importantly our results are obtained under realistic assumptions where we account for the dominant source of noise in practice. We find that in this situation we can gain nearly the full potential of entanglement estimated without accounting for decoherence. Furthermore the adaptive protocol allows for a higher Ramsey time, which gives an improvement even for uncorrelated atoms.

We gratefully acknowledge the support of the Lundbeck Foundation and the Danish National Research Foundation through QUANTOP. The research leading to these results has received funding from the European Research Council under the European Union's Seventh Framework Programme (FP/2007-2013)/ERC Grant No. 306576.

\footnotetext{
*borregaard@nbi.dk

†anders.sorensen@nbi.dk
}

[1] G. Santarelli, P. Laurent, P. Lemonde, A. Clairon, A. G. Mann, S. Chang, A. N. Luiten, and C. Salomon, Phys. Rev. Lett. 82, 4619 (1999).

[2] W. M. Itano, J. C. Bergquist, J. J. Bollinger, J. M. Gilligan, D. J. Heinzen, F. L. Moore, M. G. Raizen, and D. J. Wineland, Phys. Rev. A 47, 3554 (1993).

[3] D. J. Wineland, J. J. Bollinger, W. M. Itano, and D. J. Heinzen, Phys. Rev. A 50, 67 (1994). 
[4] J. J. Bollinger, W. M. Itano, D. J. Wineland, and D. J. Heinzen, Phys. Rev. A 54, R4649 (1996).

[5] A. André, A.S. Sørensen, and M. D. Lukin, Phys. Rev. Lett. 92, 230801 (2004).

[6] T. Rosenband, arXiv:1203.0288v2.

[7] V. Giovanetti, S. Lloyd, and L. Maccone, Nat. Photonics 5, 222 (2011).

[8] D. Leibfried, M. D. Barrett, T. Schaetz, J. Britton, J. Chiaverini, W. M. Itano, J. D. Jost, C. Langer, and D. J. Wineland, Science 304, 1476 (2004).

[9] I. D. Leroux, M. H. Schleier-Smith, and V. Vuletić, Phys. Rev. Lett. 104, 073602 (2010).

[10] J. Appel, P. J. Windpassinger, D. Oblak, U.B. Hoff, N. Kjærgaard, and E. S. Polzik, Proc. Natl. Acad. Sci. U.S.A. 106, 10960 (2009).

[11] A. Louchet-Chauvet, J. Appel, J. J. Renema, D. Oblak, N. Kjaergaard, and E. S. Polzik, New J. Phys. 12, 065032 (2010).

[12] M. F. Riedel, P. Böhi, Y. Li, T. W. Hänsch, A. Sinatra, and P. Treutlein, Nature (London) 464, 1170 (2010).

[13] C. Gross, T. Zibold, E. Nicklas, J. Esteve, and M. K. Oberthaler, Nature (London) 464, 1165 (2010).

[14] S. F. Huelga, C. Macchiavello, T. Pellizzari, A. K. Ekert, M. B. Plenio, and J. I. Cirac, Phys. Rev. Lett. 79, 3865 (1997).

[15] D. W. Berry and H. M. Wiseman, Phys. Rev. Lett. 85, 5098 (2000).

[16] B. L. Higgins, D. W. Berry, S. D. Bartlett, H. M. Wiseman, and G. J. Pryde, Nature (London) 450, 393 (2007).

[17] N. F. Ramsey, Molecular Beams (Oxford University, New York, 1956).

[18] G. Santarelli, P. Laurent, P. Lemonde, A. Clairon, A. G. Mann, S. Chang, A. N. Luiten, and C. Salomon, Phys. Rev. Lett. 82, 4619 (1999).

[19] C. W. Chou, D. B. Hume, J. C. J. Koelemeij, D. J. Wineland, and T. Rosenband, Phys. Rev. Lett. 104, 070802 (2010).

[20] E. Peik, T. Schneider, and C. Tamm, J. Phys. B 39, 145 (2006).

[21] T. L. Nicholson, M. J. Martin, J. R. Williams, B. J. Bloom, M. Bishof, M. D. Swallows, S. L. Campbell, and J. Ye, Phys. Rev. Lett. 109, 230801 (2012).
[22] A. W. Chin, S. F. Huelga, and M. B. Plenio, Phys. Rev. Lett. 109, 233601 (2012).

[23] D. J. Wineland, C. Monroe, W. M. Itano, D. Leibfried, B. E. King, and D. M. Meekhof, J. Res. Natl. Inst. Stand. Technol. 103, 259 (1998).

[24] T. Rosenband and D. R. Leibrandt, arXiv:1303.6357.

[25] J. Borregaard and A. S. Sørensen, arXiv:1304.5944.

[26] N. Shiga and M. Takeuchi, New J. Phys. 14, 023034 (2012).

[27] M. Kitagawa and M. Ueda, Phys. Rev. A 47, 5138 (1993).

[28] G. Dick, in Proceedings of the Nineteenth Annual Precise Time and Time Interval (PTTI) Applications and Planning Meeting, Redondo Beach, CA, 1987 (Naval Observatory, Washington, DC, 1987), p. 133.

[29] M. Takamoto, T. Takano, and H. Katori, Nat. Photonics 5, 288 (2011).

[30] N. Hinkley, J. A. Sherman, N. B. Phillips, M. Schioppo, N. D. Lemke, K. Beloy, M. Pizzocaro, C. W. Oates, and A. D. Ludlow, arXiv:1305.5869.

[31] J. Lodewyck, P. G. Westergaard, A. Lecallier, L. Lorini, and P. Lemonde, New J. Phys. 12, 065026 (2010).

[32] See Supplemental Material at http://link.aps.org/ supplemental/10.1103/PhysRevLett.111.090801 for (i) adaptive protocol and the various noise terms that affect the stability of the clock; (ii) details of our numerical simulations of an atomic clock.

[33] M. H. Schleier-Smith, I. D. Leroux, and V. Vuletić, Phys. Rev. Lett. 104, 073604 (2010).

[34] J. L. Sørensen, J. Hald, and E. S. Polzik, Phys. Rev. Lett. 80, 3487 (1998).

[35] K. Hammerer, K. Mølmer, E. S. Polzik, and J. I. Cirac, Phys. Rev. A 70, 044304 (2004).

[36] L.-M. Duan, J. I. Cirac, P. Zoller, and E. S. Polzik, Phys. Rev. Lett. 85, 5643 (2000).

[37] A. Kuzmich, L. Mandel, and N. P. Bigelow, Phys. Rev. Lett. 85, 1594 (2000).

[38] K. Hammerer, A. S. Sørensen, and E. S. Polzik, Rev. Mod. Phys. 82, 1041 (2010).

[39] A. S. Sørensen and K. Mølmer, Phys. Rev. Lett. 86, 4431 (2001). 